\title{
Low-intensity Irrigation Efficiency and their Application in the Conditions of Mountain Agriculture in Azerbaijan
}

\section{Aliyev $\mathbf{Z H}^{*}$}

Institute of Erosion and Irrigation, Azerbaijan National Academy of Sciences, Baku, Azerbaijan

\begin{abstract}
This article examines the issues of introduction of low-intensity irrigation systems that are justified by their efficiency in the use of the conditions of mountain agriculture in Azerbaijan. The author argues that the advantage of low-intensity irrigation technology lies in the fact that at a minimum a small amount of moisture throughout the irrigated area is formed microclimate for more intensive development of plants, thus significantly increases the yield of different types of crops, improved water distribution process ensures uniform distribution scheme concentration, etc.
\end{abstract}

Keywords: Low-intensity; Irrigation; Microclimate; Soil moisture; Yield; Impulse sprinkler; Finely divided aerosol; Sprinkler irrigation; Drip irrigation; Injection irrigation; Micro irrigation

\section{Introduction}

In conditions of acute water shortage (characteristic mainly of the mountainous and foothill zone of the republic) consumed for irrigation and cultivation of crops, the application of low-intensive environmentally friendly technologies and technical irrigation equipment, as applied to various soil and climatic conditions, becomes very important national economic importance.

The advantage of low-intensity irrigation technology is that with a minimum amount of moisture, a microclimate is formed throughout the irrigated area for more intensive plant development, and consequently, the yield of various types of crops is significantly increased. In addition, it does not require strict planning of the irrigated area (fields), allows to dose the irrigation norm, namely, at the request of crops grown in these fields, to mechanize and fully automate the process of water distribution, ensure its uniform distribution according to the concentration scheme, etc.

Low-intensity irrigation is divided into the following types of irrigation:

1. Pulse sprinkling

2. Fine (aerosol) sprinkling

3. Pulse-drop irrigation

4. Drip irrigation

5. Injection irrigation

6. Subsoil irrigation

7. Micro-irrigation (supra-crown, supra-crown and sub-root, combined, etc.)

\section{Methodology}

The technology of irrigation of fine-dispersed and micro-irrigation will be defined as impulse irrigation, and injection subsoil irrigation, as a drip irrigation. Therefore, here we are mainly concerned with the technology of pulsed as usual, and the pulse self-oscillating action, and drip irrigation. The irrigation process is characterized by the duration and intensity of the impact on distance and environment during the growing season of plant development and the daily cycle. By the degree of approximation of the intensity of water supply to the intensity of water consumption, the following types of irrigation should be distinguished:

Absolute synchronous: Water supply is carried out in full accordance with the changing intensity of water consumption during the irrigation period and the daily cycle.

Synchronous: Water supply is carried out monotonously during the day in accordance with the daily average intensity of water consumption.

Asynchronous: The intensity of water supply is greater than the instantaneous and average daily water intensity.

By type of distribution, the following types of irrigation systems are distinguished:

- Alternate irrigation by separate irrigation devices or their groups operating in a single technological cycle.

- Accumulation of water volumes in special reservoirs directly from irrigation devices or on a water supply network to ensure the technological process of irrigation.

- ensuring the continuous operation of all low-flow irrigation devices of the irrigation system. The goal of low-intensity irrigation is the creation of comfortable conditions for plants (using the example of pulse, a self-oscillating system of self-oscillating action), as well as moisture retention in the soil and partly in the ground layer of air. The immediate objects of the irrigation process are soil, plants and the ground layer of air.

Regulation of water and associated air, heat, water, and salt regime of the soil determines the development of physicochemical and biochemical processes occurring in the soil and determining its

${ }^{*}$ Corresponding author: Aliyev ZH, Institute of Erosion and Irrigation, Azerbaijan National Academy of Sciences, Baku, Azerbaijan, Tel: +994124400382; E-mail: zakirakademik@mail.ru

Received October 18, 2017; Accepted October 23, 2017; Published October 30, 2017

Citation: Aliyev ZH (2017) Low-intensity Irrigation Efficiency and their Application in the Conditions of Mountain Agriculture in Azerbaijan. Agrotechnology 6: 169, doi: 10.4172/2168-9881.1000169

Copyright: @ 2017 Aliyev ZH. This is an open-access article distributed under the terms of the Creative Commons Attribution License, which permits unrestricted use, distribution, and reproduction in any medium, provided the original author and source are credited. 
Citation: Aliyev ZH (2017) Low-intensity Irrigation Efficiency and their Application in the Conditions of Mountain Agriculture in Azerbaijan. Agrotechnology 6: 169. doi: 10.4172/2168-9881.1000169

Page 2 of 5

fertility. The stressful effects of irrigation can lead to the destruction of the structure and water resistance of soil aggregates, which reduces the fertility of the soil. Individual types of irrigation exert influence not only on the soil, but also on the surface air layer, as well as directly on the plant (regulate its water regime and photosynthetic processes, including through extra-root water supply to the aboveground part of the plant). During drip irrigation, water is distributed along the drip fields from porous humidifiers or micro-discharges mainly under the action of capillary forces. Environmental safety of irrigation for the environment should be based primarily on water-saving technologies. At the same time, it is necessary to create conditions for reducing the loss of water at the discharge and the soil surface, as well as for deep filtration in order to make fuller use of natural precipitation.

Improving the technological process of irrigation is one of the most important tasks of the scientific and technical progress of irrigated agriculture.

In this direction the great works in the republic were conducted under the guidance of the scientists G.Mammadov, B.G. Aliyev and others, also in the Russian Federation under the leadership of V.F. Nosenko, G.V. Voronin, A. Malyshev, M.P, Pisarev and others [15]. The authors believe that for the proper conduct of the irrigation technological process, it is necessary to solve a number of complex problems that meet the requirements of plants (crops). This is primarily the need to establish optimal conditions for the biological development of plants. Provision of ecologically permissible levels of soil moisture, and surface air layer, as well as aeration of soil to preserve and improve its fertility during irrigation against the background of natural little expected precipitation. Improved irrigation technology should serve to obtain guaranteed crop yields, regardless of weather conditions due to management of water and related air, thermal, salt, microbiological and nutritional regimes in the soil. The scheme of the effect of irrigation on plants and the medium is shown in Figure 1.

It should also be noted that the proper application of irrigation technology has a huge impact on crop formation. The yield of plants depends on the water factor.

To obtain the maximum yield (Minds) with a specific agrophylon and meteorological conditions for plants, comfortable conditions should be created (optimal soil moisture and surface air layer).
The influence of the necessary factors on the life of plants and on the yield increase can be expressed by the following dependence:

$$
\begin{aligned}
& Y=A P[1-(1-f i) 2] n \\
& i=1
\end{aligned}
$$

Where, $\mathrm{A}$ is the maximum gain (yield) under optimal conditions;

fi- the relative value of the i-th factor of plant life (the ratio of its actual value to the optimum);

$\mathrm{n}$ is the number of factors influencing the yield.

The effectiveness of reducing the intensity of water supply and reducing the duration of inter-irrigation periods can be assessed by comparing the yield increments.

After the transformation of expression (1) and certain assumptions, it was established that the yield increase from each of the factors under periodic irrigation, all other things being equal, is always less than the crop increment under continuous irrigation.

$$
\mathrm{Y}_{\mathrm{i}}(n e p)=\mathrm{Y}_{\mathrm{i}}(n e p)-\frac{E i^{2}(n e p) t}{l_{2}}
$$

Where, Yi (nep) is the yield increase at the mean value of the i-th factor during the irrigation cycle of traditional irrigation;

Yi (nep) - yield increase at a constant value of i-gl. factor in the process of continuous irrigation;

Ei - Relative change of the i-th factor over the meteorological period;

$\mathrm{t}$ is the duration of the meteorological period.

Analysis of the dependence (2), which reflects the quantitative assessment from the transition of periodic irrigation to continuous, shows that the difference between crop increments increases in proportion to the square of the duration of the inter-irrigation period. Thus, with the use of low-intensity irrigation technology, crop yields should be expected in comparison with traditional irrigation by maintaining the soil moisture and surface air layer at comfortable levels for plants, which is ensured by using a synchronous-impulse sprinkler system.

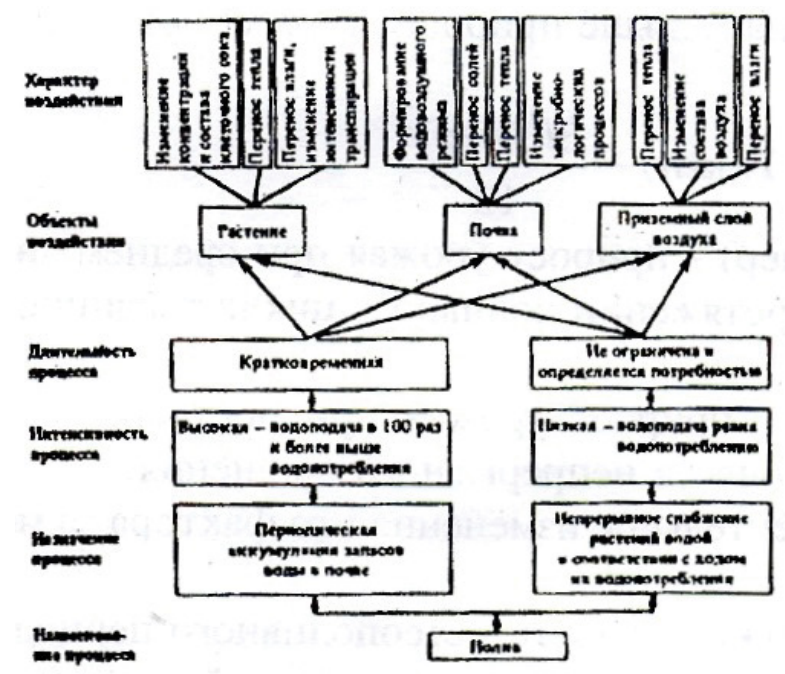

Figure 1: The scheme of the effect of watering on the plant and the environment. 
Citation: Aliyev ZH (2017) Low-intensity Irrigation Efficiency and their Application in the Conditions of Mountain Agriculture in Azerbaijan. Agrotechnology 6: 169. doi: 10.4172/2168-9881.1000169

Page 3 of 5

Features of irrigation technology with synchronous impulse sprinkling consist in the fact that the occurrence of a deficit of soil moisture in irrigated areas is compensated for by daily water supply.

The soil moisture reserves of the active layer of moisture exchange in the dry period are continuously maintained at the optimum level without the cyclicity inherent in traditional irrigation technologies. The optimal level of moisture reserves is determined depending on the type of soil and the phase of development of agricultural crops. When the moisture reserves in the active moisture exchange layer are reduced to the optimum level, sprinkling in the pulsed mode is carried out. The moisture deficit over the settlement period is calculated from the water balance equation-the water consumption of agricultural crops is determined from the approved for specific zones. At the same time, water consumption of agricultural crops is determined $b$.

$$
\mathrm{Ev}=\mathrm{Ed} \mathrm{K} 6 \mathrm{KM}
$$

Where, Ev - water consumption of agricultural crops for the billing period, $\mathrm{mm}$

Ed - the sum of the average daily air humidity deficits for the billing period, $\mathrm{Mb}$.

К6 - biological coefficient, reflecting the features of plant development.

$\mathrm{Km}$ - microclimatic coefficient, taking into account the change in the meteorological regime under the influence of irrigation.

The rate of water supply for the billing period is determined by the dependence.

$$
m=\frac{Д_{\sigma} * T * K_{c M}}{\beta} \mathcal{M}
$$

Where, DB - the average daily water consumption deficit for the billing period, $\mathrm{mm} / \mathrm{day}$;

$\mathrm{T}$ - Duration of the calculation period, days.

$\mathrm{Kcm}$ - coefficient that takes into account the costs of wetting the leaf surface of agricultural plants under pulsed sprinkling, is taken to be from 1 to 1.25 depending on the culture, development phase and the percentage of irrigated area under the crown and plant surface of plants;

$\beta$ is a coefficient that takes into account the loss of water in the zone of the rain cloud to evaporation during pulsed sprinkling.

The coefficient is determined by the formula:

$$
\beta=\frac{100-\mathrm{U}}{100}
$$

Where, $\mathrm{U}$ - evaporation of water in a zone of a rain cloud at pulse sprinkling, in\% from water supply.

Evaporation of water in a zone of a rain cloud at a pulse sprinkling is equal to:

$$
\mathrm{U}=\mathrm{t}\left(1 \frac{\alpha}{100}\right)\left(0.15 V_{p}+0.71\right) \%
$$

Where, $\alpha$ is the relative humidity of the air at the time of sprinkling, in\%;

$\mathrm{t}$ is the air temperature at the time of sprinkling, in $\mathrm{C} 0$

$\mathrm{Vp}$ - design wind speed, reduced to an altitude of 2 meters above the surface of the earth, $\mathrm{m} / \mathrm{s}$; the value of which can be determined by the following formula:

$$
\mathrm{Vp}=\mathrm{Vcp}{ }^{*} 0.7
$$

Where $\mathrm{Vcp}$ is the average wind speed over the calculation period measured at the height of the weather vane, $\mathrm{m} / \mathrm{sec}$.

To solve the process of irrigation technology, it is necessary to determine the specific flow rate.

The estimated specific consumption is determined on the basis of the compensation condition for the average daily deficit of water consumption, the cost of water for microclimate formation and demolition beyond the irrigated area by the formula:

$$
q=\frac{m}{8.64 * T * K_{c y m} \mathrm{~K}_{\mathrm{a}}}
$$

Where, $\mathrm{q}$ is the specific water flow rate $1 /$ s.ga;

$\mathrm{m}$ - Waterfall rate for the billing period, $\mathrm{mm}$;

$\mathrm{T}$ - Duration of the calculation period, day;

Ksut-coefficient of time-of-day use with round-the-clock operation of the low-intensity irrigation system, 0.95;

$\mathrm{Ka}$ is a coefficient that takes into account agrotechnical work that determines the periodic shutdown of the system (complex) operation depending on specific conditions.

Daily water supply, taking into account the cost of water for creating a microclimate ( $\mathrm{m}$ gross) and the duration of the operation of the pulse irrigation system $(\mathrm{T})$ in a simplified form can be determined by the formula:

Mbrutto - (Еисп - Kh) 10;

$$
T=\frac{m * T_{u}}{3,6 \mathrm{n} * \mathrm{~V}_{\mathrm{B}}}
$$

Where, Еисп - evaporation from a water surface for the previous day, $\mathrm{mm}$;

$\mathrm{h}$ - Atmospheric precipitation, $\mathrm{mm}$;

$\mathrm{K}$ is the coefficient of precipitation use;

M6p - daily water supply, $\mathrm{m} / \mathrm{ha}$;

Tc is the cycle time of the system, $s$;

3, 6 - conversion factor;

$\mathrm{n}$ - Number of sprinklers on one hectare, pcs / ha;

Vв - volume of a splash of a pulse sprinkler, л.

When studying the irrigation watering process, the total water consumption of plants should be determined according to the existing method, in which the water balance method is used.

$\mathrm{Ev}=\mathrm{m}+\mathrm{kh}+\Delta \mathrm{W}+\mathrm{z}$

Where, Ev - total consumption of moisture by the root layer, $\mathrm{mm}$;

$\mathrm{m}$ - The received moisture in the computational layer of soil due to irrigation during the period under consideration, $\mathrm{mm}$;

$\mathrm{k}$ is the coefficient of precipitation use.

$\mathrm{h}$ - Atmospheric precipitation, $\mathrm{mm}$.

$\Delta \mathrm{W}=\mathrm{w} 1-\mathrm{w} 2$, respectively, the moisture reserve in the soil at the beginning and end of the period under consideration, $\mathrm{mm}$. 
Citation: Aliyev ZH (2017) Low-intensity Irrigation Efficiency and their Application in the Conditions of Mountain Agriculture in Azerbaijan. Agrotechnology 6: 169. doi: 10.4172/2168-9881.1000169

Page 4 of 5

$\mathrm{z}$ - Moisture exchange with underlying layers of soil, $\mathrm{mm}$.

If the groundwater is located at a depth of more than three meters does not affect the moisture exchange of the root layer of the soil, then the component $\mathrm{Z}$ of the formula is not taken into account in subsequent calculations, i.e.

$$
\mathrm{Ev}=\mathrm{m}+\mathrm{kh}+\Delta \mathrm{W}
$$

It should be noted that the productively used precipitation for water supply is of great practical importance. To date, there is no single method for determining the productively used precipitation, which is acceptable for practical applications in the technological process of irrigation.

This part of atmospheric precipitation is taken into account in the calculation of irrigation norms and is an integral part of it, the productively used precipitation is characterized by the coefficient of precipitation use, the numerical value of which is determined by the formula:

$$
\mathrm{K}=\frac{\mathrm{W}_{2}-W_{1}+\Sigma E_{u c n}}{\mathrm{~h}}
$$

where, Эисн - evaporation from a water surface for the considered period, $\mathrm{mm}$;

W1 - Moisture reserve in the soil before precipitation, $\mathrm{mm}$;
W2- Moisture reserve in the soil after precipitation, $\mathrm{mm}$;

h - Atmospheric precipitation, $\mathrm{mm}$;

$\mathrm{K}$ is the coefficient of precipitation use.

The moisture reserve $\mathrm{W}$, before the rain, is known, determined with the previously measured soil moisture $\beta$, and the W2 moisture reserve is determined after the precipitation of soil moisture $\beta$ measured after 1-3 days after the rain. For the convenience of calculating the parameters of the technological process of crops in synchronous-pulsed and pulsed mode with a self-oscillating sprinkler, we have developed nomograms for determining the daily rate of water supply (Figure 2).

$\mathrm{h}$ - Atmospheric precipitation, $\mathrm{mm}$.

E - Evaporation from the water surface, $\mathrm{mm}$.

$\mathrm{K}$ is the coefficient of precipitation use.

\section{Conclusion}

The technological process of determining the norms and timing of irrigation based on the measurement of soil moisture after the rain is a laborious process and such a possibility can have a limited number of farms.

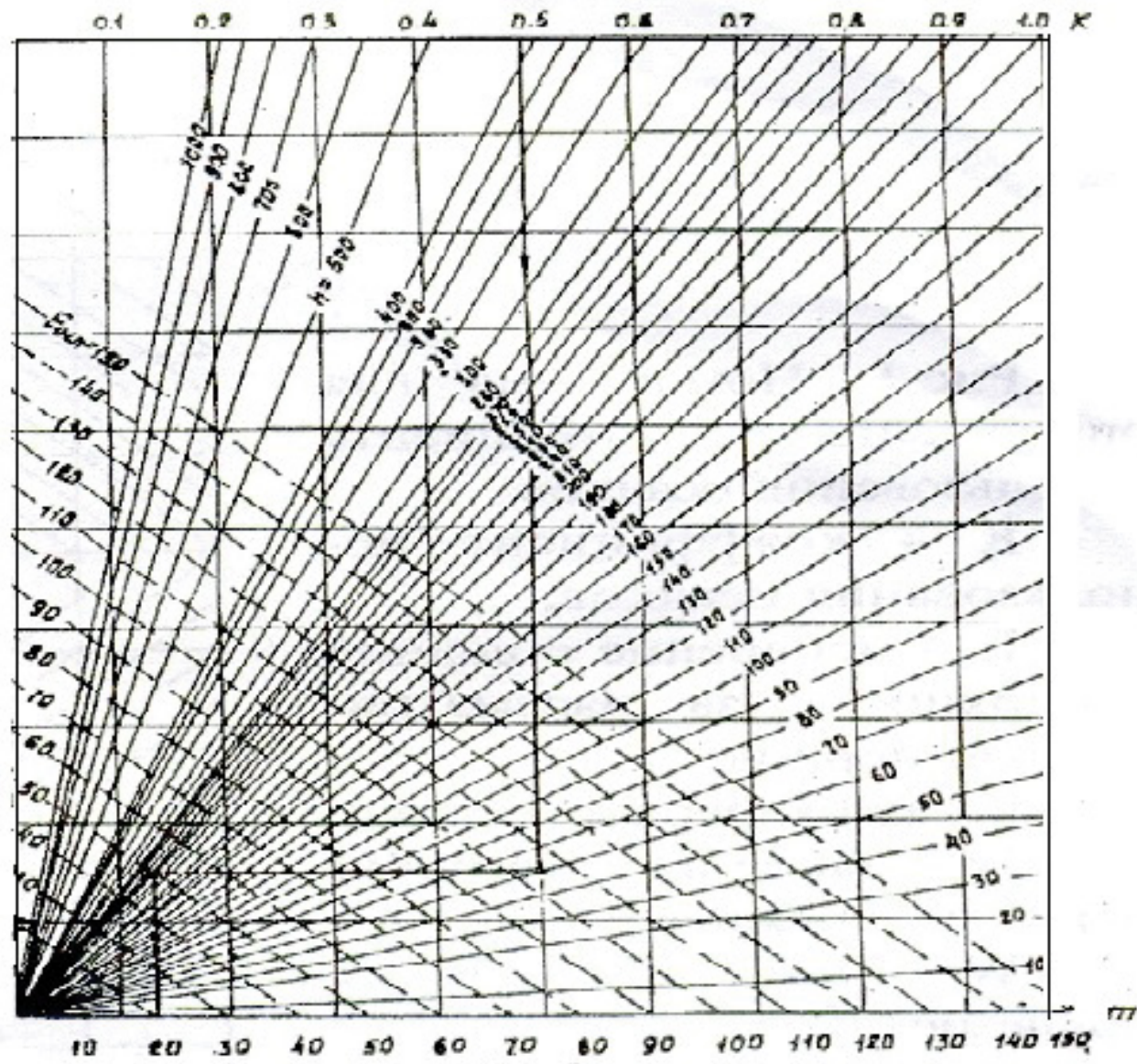

Figure 2: Nomogram for determining the irrigation norm. 
Citation: Aliyev ZH (2017) Low-intensity Irrigation Efficiency and their Application in the Conditions of Mountain Agriculture in Azerbaijan. Agrotechnology 6: 169. doi: 10.4172/2168-9881.1000169

Page 5 of 5

\section{References}

1. Aliev BH, Aliev ZH (1998) Irrigation Engineering for Farmer and Farms of Azerbaijan. Publishing house Azerneshr, Baku p: 113.

2. Aliev BH, Aliev ZH (2001) Zoning of territory of Azerbaijan Republic on choosing advanced irrigation techniques. Publishing house Ziya, Baku p: 297.

3. Aliev BH, Aliev $\mathrm{ZH}$ (2003) Irrigated agriculture in the mountain and foothill regions of Azerbaijan. Publishing house Nurlan Zia EPG Ltd, Baku p: 330.

4. Aliev BH, Aliev ZH (1999) Techniques and technology few intensive irrigations in condition of the mountain region Azerbaijan. Publishers Elm, Baku p: 220.

5. Aliev $\mathrm{BH}$, Aliev $\mathrm{ZH}$ (2007) The premises about the most important problem of the agriculture in the provision of water resources mountain and foothill regions Azerbaijan J AAS, Baku p: 179-182. 